\title{
Modulation of DNA-protein interactions in the P1 and P2 c-myc promoters by two intercalating drugs
}

\author{
Alejandro VAQUERO and José PORTUGAL \\ Departamento de Biología Molecular y Celular, Centro de Investigación y Desarrollo, CSIC, Barcelona, Spain
}

(Received 22 September 1997) - EJB 97 1358/2

\begin{abstract}
Regulation of transcription from the oncogene c-myc has an important role in the genesis of various tumors. Therefore, c-myc is a potential target for chemotherapy by drugs which are able to modify its activity directly. In this article, we identify the binding sites in the P1 and P2 promoter regions of c-myc for the intercalating antibiotics actinomycin D and elsamicin A. Gel retardation experiments indicate that actinomycin D or elsamicin A binding can inhibit the formation of several DNA-protein complexes. However, relatively low concentrations of elsamicin A, but not actinomycin D, appear to increase the level of binding to the $\mathrm{P} 1$ promoter of a protein factor. Using pure Sp1 transcription factor and an oligonucleotide containing the Sp1 putative binding site, we determined that the binding enhancement induced by small amounts of elsamicin was on the Sp1-DNA complex. Run-off transcription experiments in vitro showed that the effect of elsamicin $\mathrm{A}$ on $\mathrm{Sp} 1$ binding is followed by the maintenance or a relative rise in transcription levels from the P1 promoter of c-myc, while actinomycin D always inhibited the transcription from the $\mathrm{P} 1 \mathrm{c}-m y c$ promoter in a concentration-dependent manner. Higher concentrations of elsamicin acted as an inhibitor of the transcription from the P1 start site but not from the P2.
\end{abstract}

Keywords: c-myc; transcription; actinomycin D; elsamicin A.

The control of c-myc expression appears to be essential for normal cellular proliferation, and its failure may lead to the genesis of various tumours [1-5]. Transcription of the c-myc gene is initiated from two major start sites termed P1 and P2, which are located $161 \mathrm{bp}$ apart in the human gene [1, 2]. In normal and resting cells $\mathrm{P} 2$ is the pivotal promoter producing up to $90 \%$ of the c-myc mRNA, while P1 provides up to $25 \%$ of c-myc transcripts in normal cells, but may predominate in malignant cells $[1-3]$. Regulation has been shown to occur at multiple levels and several positive and negative cis-acting elements within a $2.3-\mathrm{kb}$ region upstream of the promoters coordinate the c-myc expression in response to various physiological stimuli [1, $6,7]$. P1 and P2 appear to be regulated by a combination of positive and negative trans-acting factors binding within this region.

The antitumor antibiotics actinomycin D (dactinomycin) and elsamicin A (elsamitrucin) $[8,9]$ are two DNA-binding drugs which intercalate preferentially into $\mathrm{G}+\mathrm{C}$-rich steps [10-14], and they are able to inhibit the transcription in vitro from prokaryotic and phage genes $[15,16]$. However, there is an increasing need to complement these studies with additional information on the effect of other antitumor drugs on specific genes, and in particular on c-myc, which plays a crucial role in malignant cell differentiation.

We have sought to verify the notion that some drugs that bind specifically to $\mathrm{G}+\mathrm{C}$-rich DNA might achieve their chemo-

Correspondence to J. Portugal, Departamento de Biología Molecular y Celular, Centro de Investigación y Desarrollo, CSIC, J. Girona-Salgado, 18-26, Barcelona, Spain

Fax: + 3432045904

E-mail:jpmbmc@cid.csic.es

Enzymes. DNase I (EC 3.1.21.1); Klenow enzyme (DNApolymerase I, large fragment) (EC 2.7.7.7); T4 polynucleotide kinase (EC 2.7.1.78). therapeutic action through their interaction with the c-myc oncogene [17-19]. In order to identify the mechanism by which some drugs inhibit the expression of the c-myc gene, we analyzed the binding of actinomycin D and elsamicin A within the region -101 to +212 of the human c-myc promoter region. We found that the presence of intercalating drugs can modulate the binding of several protein factors to the $\mathrm{P} 1$ and $\mathrm{P} 2$ promoter regions, especially the $\mathrm{Sp} 1$ trans-acting factor, which also recognizes a consensus $\mathrm{G}+\mathrm{C}$-rich tract in DNA [20]. We have also identified the binding sites for these drugs in the promoters, and show that certain discrete DNA-protein complexes behave differentially in the presence of either actinomycin D or elsamicin A since some complexes are blocked by the presence of either drug, while others seem to be insensitive, or even somewhat enhanced by the drugs. In general, impedence of the formation of DNA-protein complexes by the intercalating drugs produces the inhibition of c-myc promoter transcription in vitro, though $\mathrm{P} 1$ and P2 are differentially sensitive to the presence of drug concentrations.

\section{MATERIALS AND METHODS}

Protein extracts and drugs solutions. HeLa cell nuclear extracts were purchased from Promega; the protein concentration was between $3.6-8.3 \mu \mathrm{g} / \mathrm{ml}$ depending on the lot used. Pure Sp1 transcription factor was obtained from Promega. Actinomycin D (Serva) and elsamicin A (a gift of Dr A. M. Casazza, Bristol-Myers Squibb) were freshly prepared as $250 \mu \mathrm{M}$ stock solutions in $10 \mathrm{mM}$ Tris/ $\mathrm{HCl}, \mathrm{pH} 7.4,20 \mathrm{mM} \mathrm{KCl}$ (containing $25 \%$ methanol for elsamicin), and diluted to the desired concentrations using the same buffer without methanol prior to the experiments.

Labeling and isolation of DNA fragments. The pGEMmyc1 plasmid, which contains the SmaI-SstI fragment of the 
human c-myc [18], was kindly provided by Dr R. Ray (University of St Louis). Two restriction fragments from this plasmid were purified, SmaI-XhoI to create pVP1 and XhoI-NaeI to create pVP2, and inserted between the SmaI-SalI sites of pBluescribe (Stratagene), grown in Escherichia coli TG1 and purified using $\mathrm{CsCl}$ and standard protocols. Hence, the pVP1 plasmid contains, in its $S m a \mathrm{I}-\mathrm{XhoI}$ fragment, the c-myc region from -110 to +68 , including the P1 initiation site (fragment SX, see Fig. 1); while pVP2 contains, in its XhoI-NaeI fragment, the c-myc region from +68 to +212 including the P2 initiation site (fragment $\mathrm{XN}$, see Fig. 1). DNA fragments (SX and $\mathrm{XN}$ ) to be used in the gel retardation (band-shift) and footprinting assays were prepared by digestion with EcoRI and HindIII followed by labeling at their $3^{\prime}$-ends using $\left[\alpha-{ }^{32} \mathrm{P}\right] \mathrm{dATP}$ and the Klenow enzyme. The fragments were further separated from the remainder of the plasmids by running on a non-denaturing $6 \%$ polyacrylamide gel.

A oligonucleotide containing the consensus Sp1 binding site [20], whose sequence (upper strand) is: 5'ATTCGATCGGGGCGGGGCGAGC3' (purchased from Promega) was also employed to analyze the effect of the intercalating drugs on Sp1 binding. It was labelled at its $5^{\prime}$ ends using $\left[\gamma-{ }^{32} \mathrm{P}\right]$ ATP and T4 polynucleotide kinase.

Gel retardation assays. The gel retardation (band shift) assays were performed as described elsewhere [18] with a minor modification: the binding buffer contained $2 \%$ glycerol and $0.02 \%$ Nonidet P-40 (Sigma). In reactions containing actinomycin or elsamicin, the DNA fragments were incubated with different concentrations (see legends to figures) of the drugs before the addition of the different amounts of protein extract, except where otherwise noted. A typical reaction contained 4-12 $\mu \mathrm{g}$ HeLa extract or $25 \mathrm{ng}$ pure Sp1 protein and $1500-3000 \mathrm{cpm}$ (about $1.5 \mathrm{nmol}$ in bp) of end-labelled DNA or oligonucleotide, in the presence of $1-3 \mu \mathrm{g}$ poly[d(I-C)] (Boehringer Mannheim). The samples were analyzed on $4.5 \%$ non-denaturing polyacrylamide gels containing $45 \mathrm{mM}$ Tris/borate, $1 \mathrm{mM}$ EDTA pH 8.3. After running at low voltage $(12 \mathrm{~V} / \mathrm{cm})$, the gels were soaked in distilled water, dried under vacuum and subjected to autoradiography. Quantitative analysis of the complex formation was performed with a Molecular Dynamics computing densitometer using the ImageQuant 3.2 software. Each gel track, from four independent experiments, was normalized with respect to the total amount of loaded radioactivity and represented as the mean value \pm SD.

DNase I footprinting. Samples containing incubated complexes of the end-labelled SX or XN fragments of the human cmyc $(3000 \mathrm{cpm}$, around $10 \mathrm{pmol}$ in $\mathrm{bp})$ and different concentrations of actinomycin, or elsamicin, both in the presence and the absence of pure Sp1 transcription factor, at the concentrations stated in the legend of Fig. 7, were digested with DNase I (Boehringer Mannheim) at a final concentration of $0.01 \mathrm{U} / \mathrm{ml}$ for $2 \mathrm{~min}$ at $25^{\circ} \mathrm{C}$, and the reaction stopped by adding a solution containing $85 \%$ formamide $10 \mathrm{mM}$ EDTA and $0.02 \%$ bromophenol blue. Samples were heated at $95^{\circ} \mathrm{C}$ for $2 \mathrm{~min}$ prior to electrophoresis. The footprints were resolved by high-voltage electrophoresis in $90 \mathrm{mM}$ Tris/borate, $2 \mathrm{mM}$ EDTA pH 8.3 using $6 \%$ or $8 \%$ polyacrylamide gels containing $8 \mathrm{M}$ urea, together with a dimethylsulphate-piperidine marker specific for guanine. After running, the gels were soaked in distilled water, dried under vacuum and the bands were observed by autoradiography.

In vitro transcription. The effect of actinomycin and elsamicin on the transcription of the human c-myc P1 and P2 promoters was measured using SmaI-linearized pGEMmyc1 plasmid digested with FokI. Run-off transcription assays in vitro were performed in a final volume of $25 \mu \mathrm{l}$ and contained $30 \mathrm{mM}$ Hepes pH 7.9, $7 \mathrm{mM} \mathrm{MgCl}_{2}, 5 \mu \mathrm{M} \mathrm{ZnSO}_{4}, 1 \mathrm{mM}$ dithiothreitol,
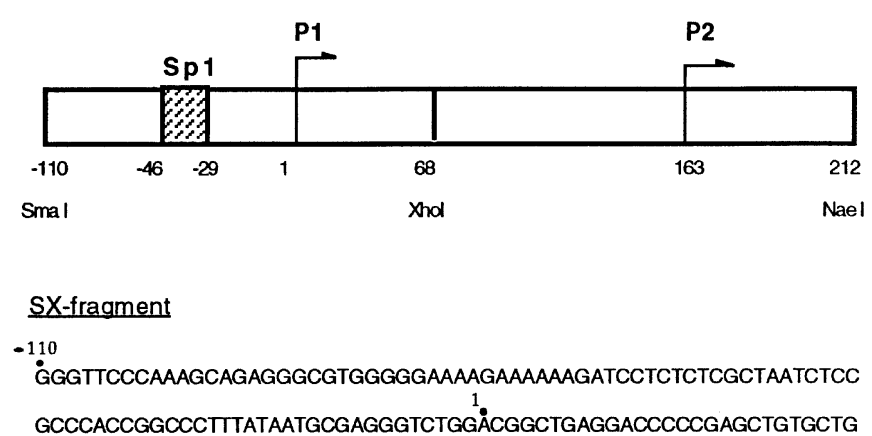

CTCGCGGCCGCCACCGCCGGGCCCCGGCCGTCCCTGGCTCCCCTCCTGCCT

68

\section{XN-fragment}

69

ĊGAGAAGGGCAGGGCTTCTCAGAGGCTTGGCGGGAAAAGAACGGAGGGAGGGATCGC

GCTGAGTATAAAAGCCGGTTTTCGGGGCTTTATCTAACTCGCTGTAGTAATTCCAGCGAG AGGCAGAGGGAGCGAGCGGGGCGGCC 212

Fig. 1. Schematic representation of the human c-myc P1 and P2 promoters. The putative Sp1 binding site, as well as the P1 and P2 start sites, are indicated (top). The indicated restriction enzymes were used to generate the two fragments: SX $(S m a I-X h o I)$ and XN (XhoI-NaeI). The lower part displays the nucleotide sequences of the upper strand of these restriction fragments.

$0.2 \mathrm{mM}$ EDTA, $2 \%$ poly(ethyleneglycol)8000 (Sigma), $300 \mu \mathrm{M}$ each of ATP, CTP and GTP, $1 \mu \mathrm{M}$ UTP and $20 \mu \mathrm{Ci}\left[\alpha^{32} \mathrm{P}\right] \mathrm{UTP}$, in the presence of the amounts of actinomycin D or elsamicin A indicated in the legend to Fig. 8. Transcription was allowed to proceed for $60 \mathrm{~min}$ at $30^{\circ} \mathrm{C}$ and the samples were phenol-extracted before gel loading. Transcription using HeLa whole nuclear extracts, in either the presence or absence of additional Sp1 transcription factor (25 ng pure protein), renders two transcripts of 348 nucleotides (P1 promoter) and 187 nucleotides ( $\mathrm{P} 2$ promoter). In the transcription experiments an internal standard for recovery and gel loading was used (a 230-nucleotide RNA kindly provided by Dr J. Bernués). Transcripts were analyzed by high-voltage electrophoresis in $90 \mathrm{mM}$ Tris/borate, 2 mM EDTA pH 8.3 using $8 \%$ polyacrylamide gels containing $7 \mathrm{M}$ urea. After running, the gels were soaked in distilled water, dried under vacuum and the bands were observed by autoradiography. The relative amounts of transcripts from both promoters, in the presence or absence of drugs, was quantified using a Molecular Dynamics computing densitometer.

\section{RESULTS}

The influence of actinomycin D and elsamicin A on the binding of protein factors, from nuclear HeLa extracts, to the human $(-110$ to +212$)$ c-myc promoter region was examined using the gel retardation technique on two restrictions fragments (designated SX and XN, see Fig. 1), which contain the P1 and $\mathrm{P} 2$ promoters respectively together with the surrounding regions. These two DNA fragments were used to identify specific protein complexes as well as their sensitivity to the intercalating ligands actinomycin D and elsamicin A.

Actinomycin D and elsamicin A bind differentially to the P2 promoter of c-myc, and compete with protein factors for their binding sites. Fig. 2 shows a gel retardation experiment using the $\mathrm{XN}$ restriction fragment of c-myc, whose sequence is displayed in Fig. 1. Four different complexes were observed 


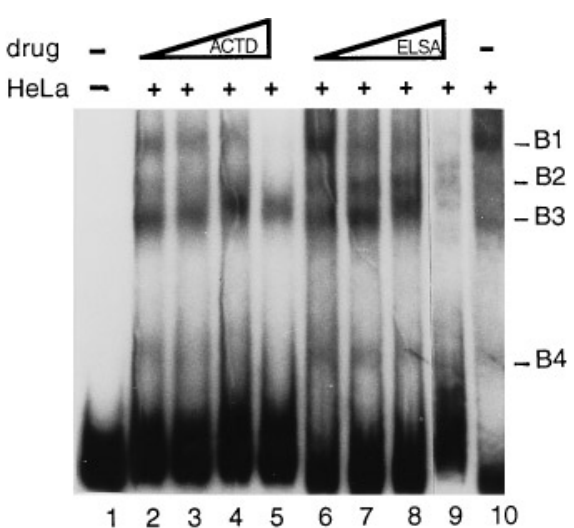

Fig. 2. Effect of actinomycin $D$ and elsamicin $A$ on the binding of a HeLa nuclear extract to the $\mathbf{P 2}$ promoter of c-myc. The experiments were performed using the $\mathrm{XN}$ restriction fragment, whose nucleotide sequence is displayed in Fig. 1. Lane 1, control DNA (1.5 nmol in bp); lanes $2-5$, gel retardation analysis of the protein binding in the presence of increasing amounts of actinomycin D (ACTD; 1, 5, 10 and $20 \mu \mathrm{M}$ ); lanes 6-9, analysis of the protein binding in the presence of increasing amounts of elsamicin A (ELSA; 5, 15, 50 and $150 \mu \mathrm{M})$; lane 10, $8 \mu \mathrm{g}$ HeLa nuclear extract plus DNA.

when this restriction fragment was incubated with HeLa extract (designated B1-B4). All of them were differentially sensitive to actinomycin and elsamicin. The presence of the B1 proteinDNA complex, the slowly migrating one in Fig. 2, was clearly inhibited by the presence of either antibiotic, though the exact concentration of drug required to inhibit the formation of the complex completely was higher in the case of elsamicin (compare lanes 5 and 9 in Fig. 2). Nevertheless, B2 and B3 were quite insensitive to the presence of either drug. In fact, concentrations of elsamicin above $100 \mu \mathrm{M}$ were required to inhibit the formation of such a complex. The most rapidly migrating complex (B4) was very sensitive to actinomycin while elsamicin was required at concentrations about $100 \mu \mathrm{M}$ to prevent the formation of this DNA-protein complex completely (compare lanes 3 and 7 in Fig. 2). A similar result, with differential modulation of DNA-protein complexes in the human c-myc $\mathrm{P} 2$ promoter, has been reported previously using mithramycin [18], a minorgroove-binding drug [21]. Several nuclear protein binding sites have been identified within the c-myc promoters [1, 2, 22]. Although our band-shift results are consistent with the presence of such binding sites, the detection of a different number of protein-DNA interactions might originate from the different protein composition or stability of the HeLa nuclear extracts, since two different preparations containing the same amount of proteins do not necessarily contain the same number of active protein factors. In any case, the behavior of the different complexes in the presence of elsamicin and actinomycin was consistent with the binding of these drugs to $\mathrm{G}+\mathrm{C}$-rich regions $[10,11]$ as it is in the experiments with mithramicin [18]. Notwithstanding, there were differences in the concentration dependence of the inhibition by the three drugs which may have arisen from a more pronounced effect of the intercalators on DNA structure, or the respective binding constants of the intercalating ligands.

At some concentrations elsamicin A might relatively enhance the binding of Sp1 protein factor to the P1 promoter of $\mathrm{c}$ $m y c$. A band shift analysis of the SX fragment, which contains the P1 start site, is displayed in Fig. 3A. At least six different protein-DNA complexes were detected when this restriction fragment was used under the experimental conditions described in Materials and Methods. They were differentially sensitive to
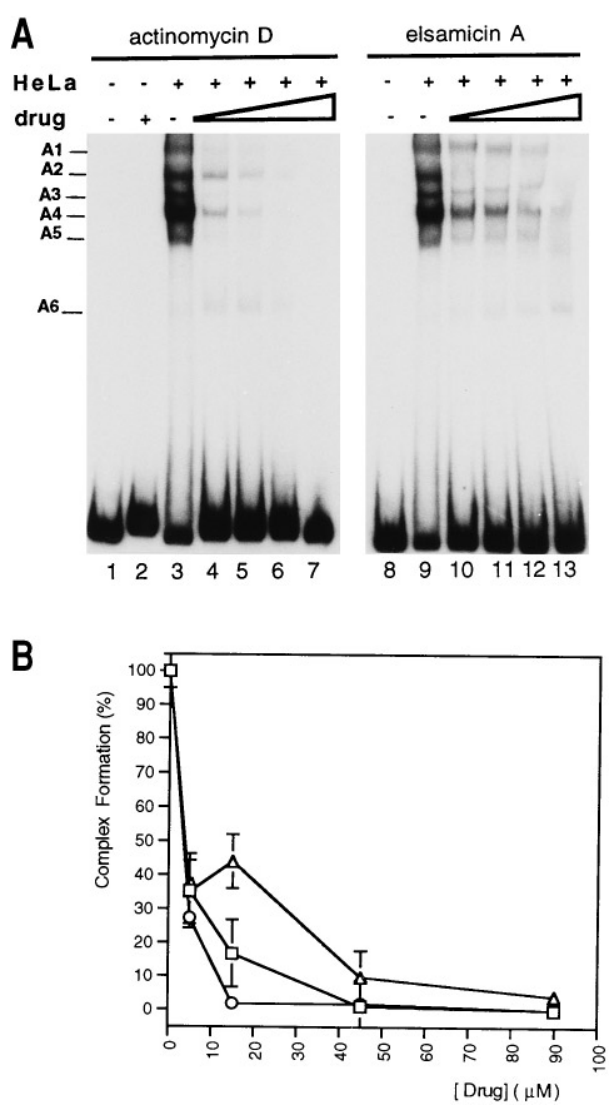

Fig.3. Analysis of the effect of actinomycin D and elsamicin A on the binding of a HeLa nuclear extract to the P1 promoter of c-myc. (A) Effect of actinomycin D and elsamicin A on the binding of a HeLa nuclear extract to the P1 promoter of c-myc (SX restriction fragment, whose nucleotide sequence is displayed in Fig. 1). Lane 1, control of DNA (1.5 pmol in bp); lanes 2 and 8, DNA plus drugs; lanes 3 and 9, DNA plus HeLa extract; lanes $4-7$, analysis of the protein binding ( $8 \mu \mathrm{g}$ of HeLa extract) in the presence of four concentrations of actinomycin $\mathrm{D}(5,15,45$ and $90 \mu \mathrm{M})$; lanes $10-13$, analysis of the protein binding in the presence of four concentrations of elsamicin $A(5,15,45$ and $90 \mu \mathrm{M})$. (B) Quantitative analysis of the effect of actinomycin D and elsamicin A on the binding of some transcription factors to the P1 promoter (displayed in A), measured as the percentage of complex formation in the presence of the drugs. $(\triangle)$ A4 complex plus elsamicin A; ( $\square$ ) A4 complex plus actinomycin $\mathrm{D}$; (O) A5 complex plus actinomycin D. The data represent the mean of four independent experiments like those in A (mean value $\pm \mathrm{SD}$ ).

elsamicin and actinomycin in a concentration-dependent manner. All the complexes were observed in the protein-DNA control experiment (lanes 3 and 9) whereas they were not observed in the control of DNA alone (lane 1). The rapidly migrating protein-DNA complex, named A5, was clearly dissociated by actinomycin $\mathrm{D}$ at concentrations above $15 \mu \mathrm{M}$, while it remained quite insensitive to elsamicin up to the same concentration. The presence of actinomycin produced a diminution in the intensity of the A2 and A4 complexes in a concentration-dependent manner. The incubation of the labelled DNA with the protein extract and either actinomycin D or elsamicin A added simultaneously also resulted in the inhibition of most of the DNA-protein complexes (results not shown). Elsamicin A produced a relative increase of the A4 band at concentrations between $5-15 \mu \mathrm{M}$ (lanes 10 and 11), although the shifted band of the complex was always less intense than in the absence of the drug (compare lanes 8 and 11 in Fig. $3 \mathrm{~A}$ ). At higher amounts of elsamicin a concentration-dependent disassociation of the DNA-protein 


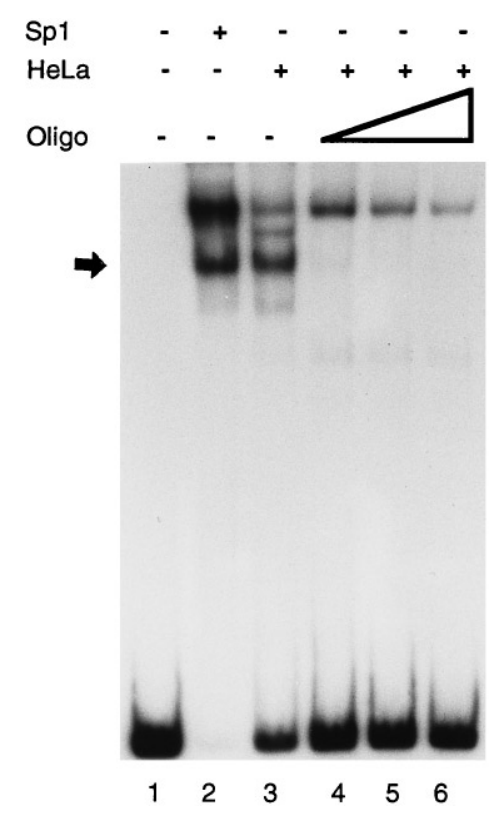

Fig. 4. Displacement of the complex A4 by increasing amounts of the oligonucleotide 5'ATTCGATCGGGGCGGGGCGAGC3' containing the Sp1 consensus sequence. The end-labelled SX fragment of c-myc was incubated with increasing amounts of unlabelled oligonucleotide, in the presence of $8 \mu \mathrm{g} \mathrm{HeLa}$ extract. Lane 1, DNA fragments; lane 2, DNA plus $25 \mathrm{ng}$ pure $\mathrm{Sp} 1$; lane 3, DNA plus HeLa extract; lanes $4-6$, $0.15,0.28$ and $0.84 \mathrm{ng}$ oligonucleotide, respectively. The band displaced by competitive binding is indicated with an arrow.

complex occurred as for actinomycin. The behavior of the A4 protein-DNA complex was reasonably reproducible in four independent experiments. The plot in Fig. 3 B shows a quantitative analysis of the complex formation in these experiments. The interference of elsamicin and actinomycin on the formation of the A4 complex was determined from densitometric scans (see Materials and Methods). The dose/response curves in Fig. 3B compare drug-treated samples with those obtained in the absence of any drug. They substantiate, within experimental error, the enhancement of the A4 band due to the binding of elsamicin, after a previous decay. A further probe of this enhancement was obtained using pure protein (see below).

To evaluate the effectiveness of both drugs as inhibitors of DNA-protein complex formation, we established conditions of the assay to maximize complex formation. The temperature for incubation and electrophoresis were optimized to enhance the stability of the DNA-protein complexes (see Materials and Methods). Due to the peculiar behavior of complex A4, we attempted to identify the relationship between this particular band and some putative protein factors that have been described to bind to this region of c-myc [2]. Fig. 4 shows a competition experiment between increased amounts of an unlabelled oligonucleotide, which contains the consensus sequence for $\mathrm{Sp} 1$ binding [20], and the SX end-labelled fragment. One of the radioactive bands, which appears to be the same as complex A4 (cf. Figs $3 \mathrm{~A}$ and 4), clearly decreased in the presence of the unlabelled competing oligonucleotide while the intensity of the other bands remained practically unaltered. This led us to identify the band, the intensity of which was modulated in direct proportion to the amount of elsamicin added, as produced by Sp1 binding (lanes $10-13$ in Fig. 3A). A putative Sp1 binding site is located upstream the P1 promoter of c-myc (see Fig. 1). It consists of two overlapping $\mathrm{Sp} 1$ binding sites, which can bind either a single $\mathrm{Sp} 1$ (giving the electrophoretic band that runs ahead) or a slowly migrating band due to the binding of two Sp1 mole-

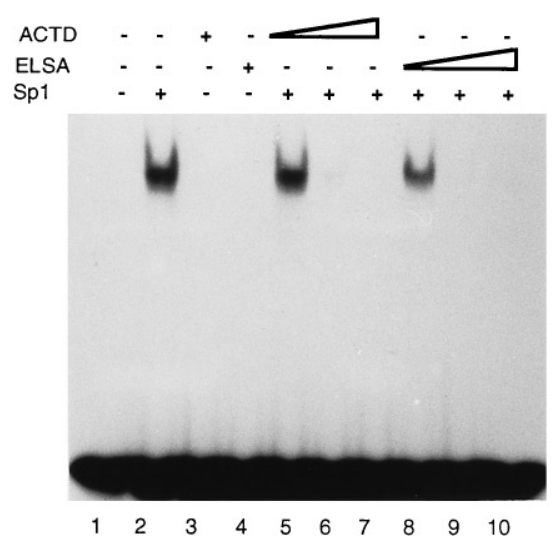

Fig.5. Effect of actinomycin D and elsamicin A on Sp1 binding to an end-labelled oligonucleotide containing its consensus binding sequence. Lanes 1-4, control lanes for DNA, protein, and drug complexes; lanes 5-7, Sp1 binding in the presence of increasing amounts of actinomycin D (ACTD; 2, 6, $15 \mu \mathrm{M}$ ); lanes $8-10$, Sp1 binding in the presence of increasing amounts of elsamicin A (ELSA; 2, 6, $15 \mu \mathrm{M}$ ).

cules (upper band in Fig. 4, lane 2). It seems that when Sp1 is displaced by the specific oligonucleotide, there is also a diminution in the intensity of the slowly migrating band, although it is not completely removed at the concentrations used to displace the A4 band. We verified our interpretation that the two bands observed should correspond to $\mathrm{Sp} 1$ binding as a monomer or homodimer complex, respectively, by the presence of a unique electrophoretic band in experiments performed with a labelled oligonucleotide containing a unique Sp1 binding site (Fig. 5).

To seek new insight into the effect of actinomycin D and elsamicin A on the binding of Sp1 to its recognition site, we performed band-shift experiments with pure Sp1 protein on both the oligonucleotide (5'-ATTCGATCGGGGCGGGGCGAGC-3', as a double strand) and the SX fragment that contains the putative $\mathrm{Sp} 1$ binding site [20] in the c-myc promoter. Illustrated in Fig. 5 are the effects of different drug concentrations on the binding of $\mathrm{Sp} 1$ to the oligonucleotide containing its consensus sequence. The retarded electrophoretic band, which corresponds to the analyzed DNA-protein complex (lane 2 in Fig. 5), was sensitive to both actinomycin and elsamicin in a concentrationdependent manner as detected by a titration of the previously formed protein-DNA complex using increasing amounts of actinomycin D (lanes 5-7) or elsamicin A (lanes 8-10). $6 \mu \mathrm{M}$ actinomycin produced more than $90 \%$ inhibition of the DNA complex, while at the same concentration, or even at smaller ones, elsamicin completely prevented the formation of an Sp1-oligo complex. These results agree with a competition between the two drugs, which are $\mathrm{G}+\mathrm{C}$ sequence-selective binding ligands $[10,23]$, and the purified $\mathrm{Sp} 1$ protein factor binding to the promoter regulatory element containing a $\mathrm{G}+\mathrm{C}$-rich tract.

At first sight, the results obtained with elsamicin A (Fig. 5), seem to be at variance with a the relative increase of the complex that was observed when total HeLa extract and the SX restriction fragment described above were employed (Fig. 3). In order to investigate the modulation of $\mathrm{Sp} 1$ binding to the c-myc promoter by elsamicin $\mathrm{A}$, and to gain further information on the effect of some antitumor antibiotics on the promoter region of c-myc, we also performed experiments with purified human Sp1 transcription factor and the SX fragment. In Fig. 6 the Sp1-DNA electrophoretic band was sensitive to actinomycin D with concentrations of around $5 \mu \mathrm{M}$ preventing the formation of DNAprotein complexes. However, while Sp1-DNA complex formation seemed to be fairly blocked around $1.5 \mu \mathrm{M}$ elsamicin A (Fig. 6B, lane 6), at higher concentrations (lanes 7-9) the 
A



B

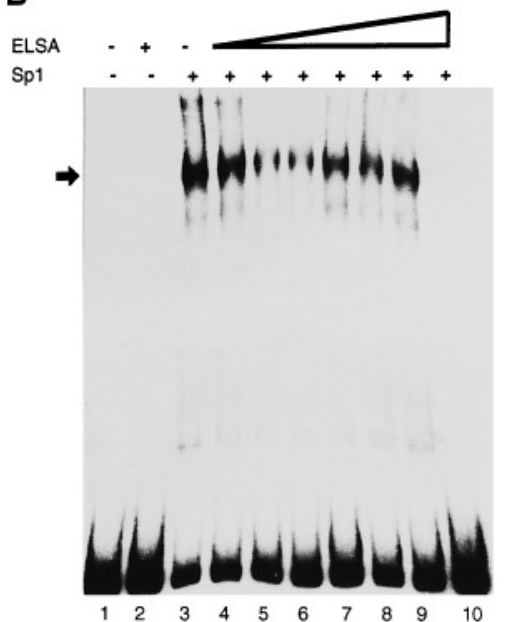

Fig. 6. Analysis of the effect of actinomycin D on Sp1 binding to the P1 promoter. (A) Effect of actinomycin D on Sp1 binding to the P1 promoter (SX restriction fragment whose nucleotide sequence is displayed in Fig. 1). Lane 1, DNA alone; lane 2, DNA plus 25 ng pure Sp1; lane 3, DNA plus $15 \mu \mathrm{M}$ actinomycin D (ACTD), lane 4, DNA plus $15 \mu \mathrm{M}$ elsamicin A (ELSA); lanes 5-9, DNA-Sp1 complex incubated with increasing concentrations of actinomycin D $(0.1,0.5,1,5$ and $15 \mu \mathrm{M})$. (B) Effect of elsamicin A on Sp1 binding to the same fragment containing the P1 promoter. Lane 1, DNA alone; lane 2, DNA plus $5 \mu \mathrm{M}$ elsamicin A; lane 3, DNA plus $25 \mathrm{ng}$ pure Sp1; lanes 4-10, DNA-Sp1 complex incubated with increasing concentrations of elsamicin $\mathrm{A}(0.5,1,1.5,2,2.5,5$ and $15 \mu \mathrm{M})$.

electrophoretic band increased slightly, in agreement with the observations using total nuclear HeLa extract described above (compare Figs 3 and 6B). There is also a clear effect on the slowly migrating band, which would correspond to the binding of two Sp1 molecules as described above. When higher concentrations (around $15 \mu \mathrm{M}$ ) were used there was an inhibition of the complex formation. These experiments were also performed using different concentrations of elsamicin and the results were unambiguously reproducible. In any case, the DNA concentration in the band shifts was about $1.5 \mathrm{nmol}$ (in bp), so that there was a molar excess of both purified $\mathrm{Sp} 1$ and drug molecules. As a result, under our experimental conditions, the amount of drug that can block (or enhance) the binding of the transcription factor to DNA (or compete for the $\mathrm{G}+\mathrm{C}$-rich zones in DNA) was limited by the equilibrium binding constant of the reaction rather than by the drug/DNA stoichiometric ratio.

Footprinting analysis showed that Sp1 and elsamicin A can compete for their overlapping binding sites. DNase I footprinting experiments were performed to characterize binding of the pure Sp1 protein to the P1 promoter of c-myc, in the presence and absence of actinomycin and elsamicin. By using this approach, it is feasible to assess the overlapping binding sites of Sp1 and either actinomycin or elsamicin A to the c-myc P1 promoter. In all respects, binding of these drugs to the SX restriction fragment, in the absence of the protein factor, produced a footprinting pattern (Fig. 7) which resembled those obtained for these antibiotics on other DNA fragments $[10,11]$. The reactions containing $\mathrm{Sp} 1$ showed a clear protection in a wide region of the P1 promoter. The presence of $5 \mu \mathrm{M}$ actinomycin D or elsamicin A produced a clear footprint in the region which overlapped the $\mathrm{Sp} 1$ footprint. We consider that either actinomycin or elsamicin can compete with the protein binding to its putative site in the c-myc P1 promoter in a concentration-dependent way. The experimental fact that elsamicin and actinomycin bind extensively to the same sites as Sp1 suggests that spacious overlapping of the binding sites is required to inhibit the binding of the protein factor, in agreement with the footprinting pattern generated by mithramycin binding to the Sp1 binding site [18]. In the

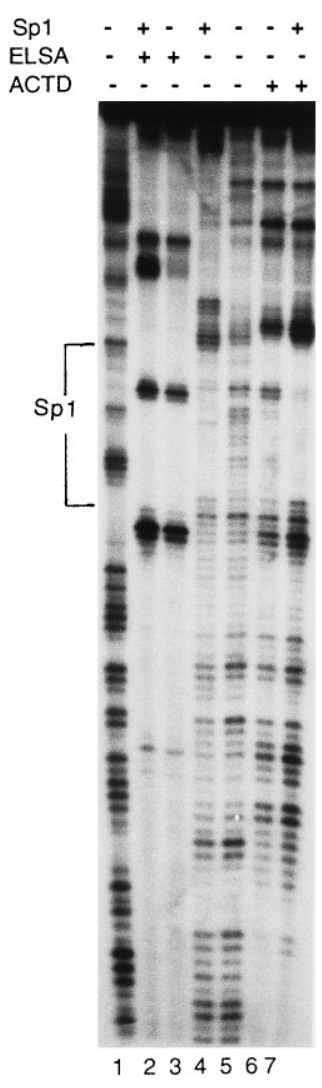

Fig. 7. DNase I footprinting of Sp1 and actinomycin D or elsamicin A binding to the P1 promoter region. Lane 1, dimethylsulphate-piperidine markers specific for guanine; lane 2, DNase I cleavage in the presence of $5 \mu \mathrm{M}$ elsamicin A (ELSA) plus $50 \mathrm{ng}$ pure human Sp1; lane 3, in the presence of $5 \mu \mathrm{M}$ elsamicin $\mathrm{A}$; lane 4 , in the presence of $150 \mathrm{ng}$ pure Sp1; lane 5, labelled DNA alone (control); lane 6, $5 \mu \mathrm{M}$ actinomycin D (ACTD); lane 7, $5 \mu \mathrm{M}$ actinomycin D plus $50 \mathrm{ng} \mathrm{Sp} 1$. The region corresponding to the $\mathrm{Sp} 1$ footprint is indicated on the figure. 


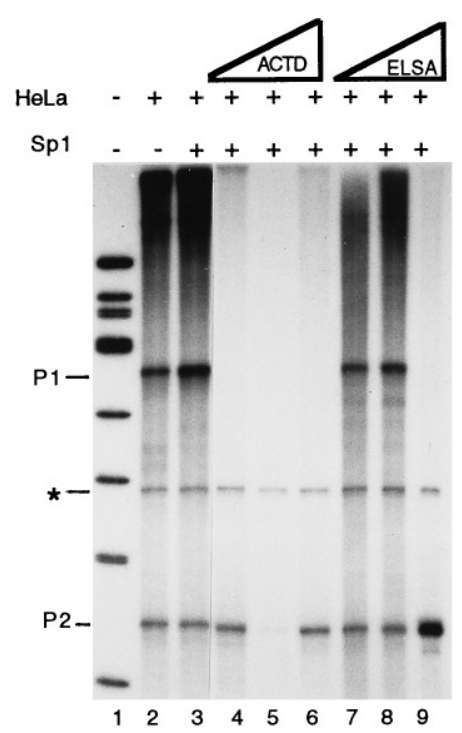

Fig. 8. Effect of actinomycin $D$ and elsamicin $A$ on in vitro run-off transcription from the c-myc $\mathrm{P1}$ and $\mathrm{P} 2$ promoters by HeLa extract. Lane 1, a size marker (end-labelled HinfI digest of $\phi X 174$ DNA) used to locate the transcripts; lane 2, transcripts from the c-myc P1 and P2 promoters (control); lane 3, transcripts obtained using whole HeLa extract plus added pure $\mathrm{Sp} 1$ (25 ng); lanes 4-6, effect of increasing amounts of actinomycin D (ACTD; 0.5, $1,5 \mu \mathrm{M}$ ) on the run-off transcription; lanes 7-9, effect of increasing amounts of elsamicin A (ELSA; $0.5,1,5 \mu \mathrm{M}$ ) on the run-off transcription. Note that in these transcription experiments an internal standard for recovery and gel loading was used (indicated by an asterisk).

footprinting studies, we did not find any symptom of increased binding of Sp1 by added elsamicin, as described in the bandshift studies (Figs 3-6) or the transcription assays in vitro, described below; undigested material is obviously difficult to observe in a region that is already protected from DNase I cleavage, though the phenomenon might exist. In Fig. 7, the differences between the DNase I cleavage enhancements in lanes 2 (elsamicin $+\mathrm{Sp} 1)$ and 7 (actinomycin $+\mathrm{Sp} 1)$ support this interpretation. An undigested region at the $3^{\prime}$ site of the c-myc P1 promoter (upper part of the gel) is observed in the presence of elsamicin A, or mithramycin [18], but not actinomycin D. It may correspond, at least in part, to a distortion of the DNA structure after $\mathrm{Sp} 1$ binding, since $\mathrm{Sp} 1$ binding can induce significant structural distortions at the $3^{\prime}$ site of G $+C$-rich regions [24]. It is worth mentioning that this region contains the TATA box of P1 (see Fig. 1), which does not coincide with a preferred binding site for the drugs studied here.

Elsamicin A changes the affinity of Sp1 protein for the P1 promoter while it can either inhibit or enhance the transcription from P2 c-myc promoter. To detect the effect of actinomycin D and elsamicin $\mathrm{A}$ on the transcription activity from the $\mathrm{P} 1$ and $\mathrm{P} 2$ promoters of c-myc in vitro, we undertook a runoff transcription assay using the pGEMmyc1 plasmid (see Materials and Methods). P1 and P2 transcripts (348 and 187 nucleotides) are clearly visible in the control (no drug added, Fig. 8, lanes 2 and 3). Lane 3 shows the result of the trancription using HeLa extract plus added Sp1 protein $(25 \mathrm{ng})$ to check the effect of elsamicin A and actinomycin D on its binding through the indirect effect on transcription of the c-myc promoter by whole HeLa extract. Lane 3 shows that adding pure Sp1 to a HeLa extract (which already contains Sp1) produced an enhancement of the amount of transcript from P1 but not from P2 promoter, as would be expected from the relative location of the Sp1 bind-



Fig.9. Quantitative analysis of the effect of elsamicin A (ELSA) or actinomycin D (ACTD) on transcription from the c-myc promoter. ( $\square$ ) P1 promoter; ( $\square$ ) P2 promoter. The bars correspond to the relative amount of transcript obtained in comparison to the basal transcription (lane 2 in Fig. 8), and considering 100\% for the c-myc transcription from $\mathrm{P} 1$. Sp1 produces a clear activation of its proximal promoter (P1, see Fig. 1) but not P2. The densitometric profiles used to generate the plot were corrected for any gel loading artifact using the band (marker) indicated by an asterisk in Fig. 8. The transcripts obtained in the presence of $1 \mu \mathrm{M}$ actinomycin D (lane 5 in Fig. 8) were not considered in this quantitative analysis since the sample was deemed to be unevenly loaded.

ing site in the c-myc promoter (see Fig. 1). Actinomycin D strongly inhibited the transcription from $\mathrm{P} 1$ at all the concentrations tested (Fig. 8, lanes 4-6) while transcription from the P2 remains almost unaltered (lane 5 in Fig. 8 should not be taken in consideration at this point since it seems to show an unevenly loaded sample). In the presence of $0.5 \mu \mathrm{M}$ elsamicin, the transcription was active from both the P1 and the P2 start sites (lane 7). From $P 1$ it even appeared to be fairly enhanced at concentrations around $1 \mu \mathrm{M}$. Higher concentrations of elsamicin were required to completely inhibit transcription from P1. Fig. 9 shows a quantitative analysis of the transcription from $\mathrm{P} 1$ and $\mathrm{P} 2$ promoters in which the amount of transcription has been normalized using a standard for recovery and loading (indicated with an asterisk in Fig. 8). The P2 transcript was clearly enhanced in the presence of $5 \mu \mathrm{M}$ elsamicin while transcription from P1 was strongly inhibited (Figs 8 and 9). These results are consistent with the general inhibition, by actinomycin $\mathrm{D}$, of c-myc transcription from the $\mathrm{P} 1$ promoter in a concentration-dependent manner. They also agree with the relative increment of Sp1 binding in the presence of some concentrations of elsamicin A, described in the band-shift assays and the correct assembly of the initiation complex on the P1 promoter at low concentrations of elsamicin $(1 \mu \mathrm{M})$. These might result in the maintenance of transcription levels and a slight increase in the yield of total transcript formed from the P1 start site (see the quantitative analysis in Fig. 9) but not from the P2 start site, which is further from the Sp1 binding site than the P1 promoter [2]. Surprisingly, once P1 is inhibited (Fig. 8, lane 9) there is a manifest increase in transcript yield from the $\mathrm{P} 2$ promoter. Tentatively, we suggest that this situation arises from a redistribution of some basal trans-acting factors due to the stronger binding of the drug in a region close to $\mathrm{P} 1$. Some effects of drugs in the relative formation of multimeric protein complexes have been described, as for example some intercalators that affect the interaction between a growth response factor and the TATA-box binding protein [25]. 


\section{DISCUSSION}

While, in general, the major groove of DNA is recognized by many, but not all, trans-acting protein factors, the minor groove is employed by several drugs, including many intercalating agents which are known to exert antitumor activity. The experimental approaches presented in this article indicate that two such intercalators, actinomycin D and elsamicin A, might induce a specific release of some proteins which selectively bind to the human c-myc promoter. Hence, it is feasible to inhibit the binding of regulatory proteins to DNA using small ligands [26, 27]. This effect may be responsible for the differential modulation of the transcription of a particular gene by intercalating agents (c-myc should be considered an example).

By exploring the band-shift assays (Figs 2 to 6) and the DNase I footprinting (Fig. 7), we can observe several DNA-protein complexes that can be completely inhibited or/and dissociated. The results presented in this article accord with the view that low-molecular-mass ligands are able to displace proteins bound to DNA [25-28]. A possible explanation is that the competition observed is caused by a change in the local DNA conformation. This may be mediated by the unwinding of the polynucleotide induced by the intercalation. Nevertheless, it is worth noting that minor-groove binding ligands, which are considered to distort DNA to a lesser extent, can also inhibit the formation of DNA-protein complexes [22, 28].

Experiments using pure $\mathrm{Sp} 1$, and either the $\mathrm{P} 1$ promoter or a consensus oligonucleotide, indicate that actinomycin $\mathrm{D}$ and elsamicin A overlap the $\mathrm{Sp} 1$ binding site, thus preventing protein binding (at any actinomycin concentration and relatively high concentrations of elsamicin). Under certain concentration-dependent conditions, the protein binding to the preferred sequence is maintained and sometimes it appears to be relatively enhanced in the vicinity of the P1 promoter. This enhancement is not observed when $\mathrm{Sp} 1$ is bound to a synthetic oligonucleotide that lacks the flanking sequences in the c-myc promoter. Since DNase I footprinting establishes that both drugs bind preferentially to $\mathrm{C}+\mathrm{G}$-rich regions in DNA $[10,11]$ including the c-myc promoters (see Fig. 7), the relative enhancement in the Sp1DNA complex in Figs 3 and 6 (in the presence of some concentrations of elsamicin, but not in the presence of any concentration of actinomycin) deserves further discussion. We tentatively consider that elsamicin A intercalates at DNA regions located within or in the vicinity of the Sp1 binding site using the unoccupied minor groove and changing DNA to a more-favored conformation for $\mathrm{Sp} 1$ binding, while actinomycin might occupy all the $\mathrm{C}+\mathrm{G}-$ rich regions and perhaps other weaker binding sites [14], thus making some of the DNA-protein complexes more sensitive than others. The binding of elsamicin is somewhat driven by the flanking sequences since the phenomenon is not observed on the consensus oligonucleotide (Figs. 5 and 6). The conformational change produced by elsamicin binding could increase the affinity constant of the Sp1 protein for its consensus sequence. In the P1 promoter of c-myc there are two overlapping sequences with eight bases identical with the consensus sequence $[2,18]$. The ability of elsamicin A to form a ternary complex, with Sp1 and the P1 promoter DNA, may lead to the stability of the protein-DNA complex at moderate drug concentrations, but not at higher ones. The run-off transcription experiments (Figs 8 and 9) agree with such an interpretation. There was a subtle sustenance of the amount of transcript from P1 in the presence of low elsamicin concentration, but not of actinomycin. In any case, the yield of transcript from P1 was below the amounts obtained without any drug. Additional experiments, using other promoters, will be required to check whether this behavior is widely observable or due only to the peculiar charac- teristics of the c-myc P1 promoter or elsamicin A. However, since we added some pure $\mathrm{Sp} 1$ in the run-off experiments, it was unambiguously established that the changes in the yield of RNA from P1 were intimately related to the levels of Sp1 binding to the P1 promoter region (see the quantitative analysis in Fig. 9). At the same time, there was a general inhibition of the transcription due to the binding of the drugs to other $\mathrm{C}+\mathrm{G}$-rich regions. Several lines of evidence also show that elsamicin and actinomycin might behave differentially in transcription from phage genes while still acting as transcription inhibitors [16]. The effects of actinomycin and elsamicin are not due to their direct interaction with the $\mathrm{Sp} 1$ protein alone, as indicated by incubating drug and protein together first (details not reported).

In the run-off transcription experiments using human c-myc (Fig. 8), a slight increment of transcripts from P1 at $1 \mu \mathrm{M}$ elsamicin is evident (after inhibition at lower concentrations), in agreement with the effect mediated through $\mathrm{Sp} 1$ recognition of its consensus sequence observed in the band-shift assays. Nevertheless, the drug somewhat retains the transcription to a level similar to that the transcription obtained using HeLa extract alone, but without reaching the amounts for the HeLa+Sp1 (lanes 2 and 3 in Fig. 8). It is likely that the binding of elsamicin to other $\mathrm{C}+\mathrm{G}$-rich sites in the promoter acts as an inhibitor of transcription, as observed with actinomycin (Fig. 8, lanes 4-6). Hence, the effect on Sp1 binding is partially masked, yet it is quantitatively evident in Fig. 9. The abilities of small ligands to both augment and arrest transcription by RNA polymerase II in a concentration-dependent fashion does not seem to be a peculiarity of the drugs and/or promoters used in this article, since the ability of some intercalating drugs as well as minor groove binders to enhance and/or impede RNA elongation has been described elsewhere $[19,28,29]$. Notwithstanding, with elsamicin the inhibition of the transcription from P1 was concomitant with the increase in transcript yield from P2, which might be explained by considering that the some protein factors released from the $\mathrm{P} 1$ promoter bind to the $\mathrm{P} 2$ promoter, thus activating the initiation from the P2 start site (Fig. 8, lane 9, and Fig. 9). Nevertheless, our results point out the occurrence of this dual effect in the transcription of an oncogene in the presence of clinically useful drugs under physiologically relevant concentrations.

So far as our results go, it seems likely that the concentration-dependent response of c-myc promoter to elsamicin A is mediated by changes in the affinity of Sp1. The concentrations of elsamicin required to observe the relative enhancement of protein binding (Fig. 3) or to increase the transcription yield (Fig. 8) are fairly different. They possibly reflect the distinct experimental conditions in the band-shift and run-off transcription experiments. RNA polymerase II needs to read through natural pause sites, in which the polymerase proceeds slowly [7, 30]. Elsamicin might act by allowing these pauses to be overridden. This explanation is consistent with the role assigned to the levels of Sp1 in eliminating the promoter proximal pausing of RNA polymerase in c-myc [7]. A direct link between the capacity of elsamicin to modulate c-myc expression and to influence biological processes will require further experiments in vivo. The results described here represent a new step towards the design of such experiments.

We are thankful to Dr J. Bernués for helpful discussions and Dr R. Ray (University of St Louis) for a gift of pGEMmyc1. Elsamicin A was kindly provided by Dr A. M. Casazza (Bristol-Myers Squibb). This work was financed by grants from the Spanish Dir. Gen. Enseñanza Superior (PB 95-0064) and Dir. Gen. de Investigación Científica y Técnica (PB93/ 102). The support of the Generalitat de Catalunya through its Centre de Referencia en Biotecnologia is also acknowledged. 


\section{REFERENCES}

1. Hay, N., Bishop, M. \& Levens, D. (1987) Regulatory elements that modulate expression of human c-myc, Genes \& Dev. 1, 659-671.

2. Marcu, K. B., Bossone, S. A. \& Patel, A. J. (1992) myc function and regulation, Annu. Rev. Biochem. 61, 809-860.

3. Arcinas, M. \& Boxer, L. M. (1994) Differential protein binding to the c-myc promoter during differentiation of hematopoietic cell lines, Oncogene 9, 2699-2706.

4. Dufort, D. \& Nepveu, A. (1994) The human cut homeodomain protein represses transcription from the c-myc promoter, Mol. Cell. Biol. 14, 4251-4257.

5. Ryan, K. M. \& Birnie, G. D. (1996) Myc oncogenes: the enigmatic family, Biochem. J. 314, 713-721.

6. DesJardins, E. \& Hay, N. (1993) Repeated CT elements bound by zinc finger proteins control the absolute and relative activities of the two principal human c-myc promoters, Mol. Cell. Biol. 13, $5710-5724$.

7. Krumm, A., Hickey, L. B. \& Groudine, M. (1995) Promoter-proximal pausing of RNA polymerase II defines a general rate-limiting step after transcription initiation, Genes \& Dev. 9, 559-572.

8. Allen, S. L., Schacter, L. P., Lichtman, S. M., Bukowski, R., Fusco, D., Hensley, M., O'Dwyer, P., Mittelman, A., Rosenbloom, B. \& Huybensz, S. (1996) Phase II study of elsamitrucin (BMY-28090) for the treatment of patients with refractory/relapsed non-Hodgkin's lymphoma, Invest. New Drugs 14, 213-217.

9. Hassan, H. T. \& Rees, J. K. (1989) Low concentrations of cytosine arabinoside, 6-thioguanine, actinomycin-D and aclacinomycin A stimulates the differentiation of normal human marrow myeloid progenitor cells, Med. Oncol. Tumor. Pharmacother. 6, 213-217.

10. Fox, K. R. \& Waring, M. J. (1984) DNA structural variations produced by actinomycin and distamycin as revealed by DNase I footprinting, Nucleic Acids Res. 12, 8271-8285.

11. Salas, X. \& Portugal, J. (1991) Map of chartreusin and elsamicin binding sites on DNA, FEBS Lett. 292, 223-228.

12. Alhambra, C., Luque, F. J., Portugal, J. \& Orozco, M. (1995) Molecular dynamics study of the binding of elsamicin A to DNA, Eur J. Biochem. 230, 555-566.

13. Rodríguez-Campos, A., Azorín, F. \& Portugal, J. (1996) Influence of elsamicin A on the activity of mammalian topoisomerase I, Biochemistry 35, 11177-11182.

14. Fletcher, M. C. \& Fox, K. R. (1996) Dissociation kinetics of actinomycin D from individual GpC sites in DNA, Eur. J. Biochem. 237, 164-170.

15. Phillips, D. R., White, R. J., Trist, H., Cullinane, C., Dean, D. \& Crothers, D. M. (1990) New insight into drug-DNA interactions at individual drug binding sites probed by RNA polymerase during active transcription of the DNA, Anti-Cancer Drug Des. 5, $21-29$.

16. Portugal, J. (1995) Abortive transcription of the T7 promoter induced by elsamicin A, Anti-Cancer Drug Des. 10, 427-438.

17. Bunch, R. T., Povirk, L. F., Orr, M. S., Randolph, J. K., Fornari, F. A. \& Gewirtz, D. A. (1994) Influence of amsacrine (m-AMSA) on bulk and gene-specific DNA damage and c-myc expression in MCF-7 breast tumor cells, Biochem. Pharmacol. 47, 317-329.
18. Snyder, R. C., Ray, R., Blume, S. \& Miller, D. M. (1991) Mithramycin blocks transcriptional initiation of the c-myc P1 and P2 promoters, Biochemistry 30, 4290-4297.

19. Fornari, F. A., Jarvis, W. D., Grant, S., Orr, M. S., Randolph, J. K., White, F. K. H. \& Gewirtz, D. A. (1996) Growth arrest and nonapoptotic cell death associated with the suppression of c-myc expression in MCF-7 breast tumor cells following acute exposure to doxorubicin, Biochem. Pharmacol. 51, 931-940.

20. Briggs, M. R., Kadonaga, J. T., Bell, S. P. \& Tjian, R. (1986) Purification and biochemical characterization of the promoter-specific transcription factor Sp1, Science 234, 47-52.

21. Keniry, M. A., Banville, D. L., Simmonds, P. M. \& Shafer, R. (1993) Nuclear magnetic resonance comparison of the binding sites of mithramycin and chromomycin on the self-complementary oligonucleotide d(ACCCGGGT)2. Evidence that the saccharide chains have a role in sequence specificity, J. Mol. Biol. 231, 753-767.

22. Chaudhary, D. \& Miller, D. M. (1995) The c-myc promoter binding protein (MBP-1) and TBP bind simultaneously in the minor groove of the c-myc P2 promoter, Biochemistry 34, 3438-3445.

23. Párraga, A. \& Portugal, J. (1992) Detection of elsamicin-DNA binding specificity by restriction enzyme cleavage, FEBS Lett. 300, $25-29$.

24. Kuwahara, J., Yonezawa, A., Futamura, M. \& Sugiura, Y. (1993) Binding of transcription factor Sp1 to GC box DNA revealed by footprinting analysis: different contact of three zinc fingers and sequence recognition mode, Biochemistry 32, 5994-6001.

25. Chiang, S.-Y., Welch, J. J., Rauscher, F. J. \& Beerman, T. A. (1996) Effect of DNA-binding drugs on early growth response factor-1 and TATA box-binding protein complex formation with the herpes simplex virus latency promoter, J. Biol. Chem. 271, 2399924004

26. Welch, J. J., Rauscher, F. J. \& Beerman, T. A. (1994) Targeting DNA-binding drugs to sequence-specific transcription factorDNA complexes. Differential effects of intercalating and minor groove binding drugs, J. Biol. Chem. 269, 31051-31058.

27. Bianchi, N., Passadore, M., Rutigliano, C., Feriotto, G., Mischiati, C. \& Gambari, R. (1996) Targeting of the Sp1 binding sites of HIV-1 long terminal repeat with chromomycin-disruption of nuclear factor. DNA complexes and inhibition of in vitro transcription, Biochem. Pharmacol. 52, 1489-1498.

28. Bellorini, M., Moncollin, V., Dincalci, M., Mongelli, N. \& Mantovani, R. (1995) Distamycin a and tallimustine inhibit TBP binding and basal in vitro transcription, Nucleic Acids Res. 23, 16571663.

29. Mote, J., Ghanouni, P. \& Reines, D. (1994) A DNA minor groovebinding ligand both potentiates and arrests transcription by RNA polymerase II. Elongation factor SII enables readthrough at arrest sites, J. Mol. Biol. 236, 725-737.

30. Plet, A., Eick, D. \& Blanchard, J. M. (1995) Elongation and premature termination of transcripts initiated from c-fos and c-myc promoters show dissimilar patterns, Oncogene 10, 319-328. 